\title{
MR Diffusional Kurtosis Imaging-Based Assessment of Brain Microstructural Changes in Patients with Moyamoya Disease before and after Revascularization
}

\author{
(D) P.-G. Qiao, (D) X. Cheng, (D) G.J. Li, (DP. Song, (D). Han, and (D) Z.-H. Yang
}

\begin{abstract}
BACKGROUND AND PURPOSE: Conventional imaging examinations are insufficient to accurately assess brain damage in patients with Moyamoya disease. Our aim was to observe brain microstructural changes in patients with Moyamoya disease by diffusional kurtosis imaging and provide support data for application of this technique in individualized assessment of disease severity and surgical outcome among patients with Moyamoya disease.
\end{abstract}

MATERIALS AND METHODS: A total of 64 patients with Moyamoya disease and 15 healthy volunteers underwent diffusional kurtosis imaging, and a second scanning was offered to surgical patients 3-4 months after revascularization. The diffusional kurtosis imaging parameter maps were obtained for mean kurtosis, axial kurtosis, radial kurtosis, fractional anisotropy, mean diffusivity, axial diffusivity, and radial diffusivity. The parameter values were measured in sensory pathway-related regions for all subjects. Differences in diffusional kurtosis imaging parameters of these brain regions were examined for healthy volunteers, patients without acroparesthesia, and asymptomatic and symptomatic sides of patients with acroparesthesia. Changes in diffusional kurtosis imaging parameters of patients with Moyamoya disease before and after revascularization were compared.

RESULTS: Compared with healthy volunteers, patients with Moyamoya disease showed decreased mean kurtosis, axial kurtosis, radial kurtosis, and fractional anisotropy in the corona radiata. Similarly, mean kurtosis, radial kurtosis, and fractional anisotropy decreased in the posterior limb of the internal capsule, whereas axial kurtosis decreased and radial kurtosis increased in the thalami of patients with Moyamoya disease compared with healthy volunteers. Compared with the asymptomatic contralateral hemisphere, the symptomatic group showed increased mean kurtosis in the contralateral primary somatosensory cortex, increased fractional anisotropy in the contralateral corona radiata and posterior limb of the internal capsule, and decreased axial kurtosis in the contralateral thalamus. Among patients with Moyamoya disease with acroparesthesia, mean kurtosis decreased in the primary somatosensory cortex on the operated side following revascularization.

CONCLUSIONS: The diffusional kurtosis imaging technique is applicable to patients with Moyamoya disease for detecting brain microstructural changes in white and gray matter before and after revascularization; this feature is useful in the assessment of disease severity and surgical outcome.

ABBREVIATIONS: $A D=$ axial diffusivity; $A K=$ axial kurtosis; DKI = diffusional kurtosis imaging; EDAS = encephaloduroarteriosynangiosis; FA = fractional anisotropy; $\mathrm{MD}=$ mean diffusivity; $\mathrm{MK}=$ mean kurtosis; $\mathrm{MMD}=$ Moyamoya disease; $\mathrm{RK}=$ radial kurtosis; RD = radial diffusivity; $\mathrm{SI}=$ primary somatosensory cortex; TTP = time-to-peak

oyamoya disease (MMD) is a chronic occlusive cerebrovascular disease characterized by stenosis and occlusion of the terminal portion of the bilateral internal carotid arteries

Received February 12, 2019; accepted after revision December 10.

From the Department of Radiology (P.-G.Q., X.C., Z.-H.Y.), Beijing Friendship Hospital, Capital Medical University, Beijing, China; Departments of Radiology (P.-G.Q., G.-J.L.) and Neurosurgery (G.-J.L., C.H.), Affiliated Hospital of Academy of Military Medical Sciences, Beijing, China; Department of Radiology (P.S.), Liaocheng People's Hospital, Liaocheng, China; and Beijing Universal Medical Imaging Diagnostic Center (G.J.L.), Beijing, China.

P.-G. Qiao and X. Cheng contributed equally to this work.

This work was supported by grants from the National Natural Science Foundation of China (No. 81701663) associated with the formation of an abnormal vascular network at the cerebral basal ganglia. ${ }^{1}$ On the basis of angiographic manifestations, Suzuki and Takaku ${ }^{2}$ classified the development of MMD into 6 stages. Currently, Suzuki staging is the primary criterion for clinical assessment of disease severity in patients with MMD. However, the natural course and prognostic factors of MMD are

Please address correspondence to Zheng-Han Yang, MD, Department of Radiology, Beijing Friendship Hospital, Capital Medical University, 95 YongAn Rd, Beijing, 100050, P.R. China; e-mail: cjr.yangzhenghan@vip.163.com

- Indicates open access to non-subscribers at www.ajnr.org

http://dx.doi.org/10.3174/ajnr.A6392 
still unclear, and its clinical manifestations vary among patients. Even patients in the same Suzuki stage may manifest different clinical features. ${ }^{3}$ In addition, the detection rate of asymptomatic MMD has been growing year by year, which further perplexes MMD treatment. ${ }^{4}$ In a multicenter study conducted in Japan, $75 \%$ of the affected hemispheres in asymptomatic patients with MMD were classified into Suzuki stages III-IV, and $~ 45 \%$ of the affected hemispheres had decreased cerebral vascular reactivity or cerebral blood flow, whereas $20.8 \%$ of the affected hemispheres had cerebral infarction lesions. ${ }^{5}$ Patients with MMD may have different clinical manifestations in long-term chronic ischemic conditions even if the Suzuki stage is identical and no changes in brain parenchyma are detected by conventional MR imaging. ${ }^{6}$ Therefore, conventional imaging examinations are insufficient to accurately assess brain damage in patients with MMD.

DWI-based DTI and diffusional kurtosis imaging (DKI) can illustrate fine changes in the microstructure of the central nervous system and quantitatively assess the damage to brain tissue. On the basis of a Gaussian distribution model, DTI can reflect microstructural changes in cerebral white matter and detect its integrity because DTI parameter changes are sensitive indicators for structural changes in axons, myelin, and organelles. ${ }^{7-9}$ This technique can be used to detect changes in early ischemic damage for patients with $\mathrm{MMD},{ }^{10}$ among whom such early damage may be associated with neurologic impairment. ${ }^{11}$ However, DTI is not sensitive to changes in cerebral gray matter due to the distribution and diffusion isotropy. On the basis of a non-Gaussian distribution model, DKI can sensitively reflect microstructural changes in gray matter areas, neurodegenerative diseases, tumor microenvironment, and posttraumatic brain tissue. ${ }^{7,12-14}$ The use of DKI enables the detection of more extensive brain damage in patients with MMD, especially early damage to the intersection of white matter fibers. ${ }^{8}$

Despite early detection of white matter microstructural damage in patients with MMD by DTI and DKI, the association between such damage and neurologic impairment in these patients remains unclear. While a handful of studies have suggested an association between this damage and cognitive impairment, its relationship with MMD and other common sensory, motor, verbal, and visual impairments is yet to be reported. In addition, there is a dearth of studies on early damage to white matter and patient prognosis, as well as the associated changes following revascularization. Moreover, DKI allows detection of gray matter microstructural changes, yet such changes have received far less investigation in patients with MMD.

In this study, we used DKI to detect microstructural changes in sensory pathway-related regions of brain tissue among patients with MMD with acroparesthesia and clarified whether such changes would improve in these patients following revascularization. The results could provide support for the application of DKI in individualized assessment of disease severity and surgical outcome among patients with MMD.

\section{MATERIALS AND METHODS}

\section{Subjects}

Sixty-four patients with MMD (27 men and 37 women) were recruited from July 2015 to April 2016. The patients had a median age of 35 years (range, 16-45 years). Thirty-three patients presented with unilateral paroxysmal numbness or hypoesthesia, 15 of whom underwent encephaloduroarteriosynangiosis (EDAS). Acroparesthesia was absent in the remaining 31 patients, 14 of whom underwent EDAS.

The inclusion criteria were as follows: 1) The patient was diagnosed and staged by digital subtraction angiography; 2) the patient remained in a stable condition, had no cerebral hemorrhage before scanning, presented with no cerebral infarction in the bilateral primary somatosensory cortex (SI) by conventional MR imaging, and cooperated with the examination; 3) the patient reported no other neurologic or psychiatric diseases; and 4) the patient was right-handed.

In addition, 15 healthy age- and sex-matched volunteers were selected. All subjects were provided with details of the examination and the necessary precautions. Signed informed consent was obtained from each subject before the examination. The ethics committee of the Affiliated Hospital of Academy of Military Medical Sciences approved the study.

\section{MR Imaging}

The MR imaging was performed on a 3T Skyra MR imager (Siemens, Erlangen, Germany) using a 32-channel standard head coil. All subjects underwent DKI, whereas patients with MMD also underwent contrast-enhanced DSC MR imaging. MR imaging was performed for all patients within 1 week before and after digital subtraction angiography, and a second MR imaging was offered to 29 surgical patients 3-4 months following EDAS.

DKI scans were acquired by axial echo-planar imaging using the following parameters: $\mathrm{TR}=5600 \mathrm{~ms}$; $\mathrm{TE}=92 \mathrm{~ms}$; FOV $=$ $228 \mathrm{~mm}$; matrix $=384 \times 384 \mathrm{~mm}^{2}$; section thickness $=3 \mathrm{~mm}$; b-value $=0,1000$, and $2000 \mathrm{~s} / \mathrm{mm}^{2}$; direction of diffusion-sensitive gradient field applied $=30$; and scanning time $=7$ minutes 2 seconds.

DSC-MR imaging was performed by echo-planar imaging using the following parameters: $\mathrm{TR}=1870 \mathrm{~ms}$; $\mathrm{TE}=30 \mathrm{~ms}$; FOV $=220 \mathrm{~mm}$; matrix $=128 \times 128 \mathrm{~mm}^{2}$; section thickness $=$ $4 \mathrm{~mm}$; section spacing $=1.2 \mathrm{~mm}$; section number $=24$; and acquisition time phase $=60$ seconds. After the start of the scan, a high-pressure syringe was used to inject a gadoliniumbased contrast agent $(0.2 \mathrm{~mL} / \mathrm{kg}$; gadopentetate dimeglumine, Magnevist; Bayer HealthCare Pharmaceuticals, Wayne, New Jersey) at the rate of $4-5 \mathrm{~mL} / \mathrm{s}$ in the sixth time phase.

\section{DKI Analysis}

Data were exported from the workstation and converted to the NIfTI1 data format using "dcm2niigui" in MRIcron (Version 6; https://www.nitrc.org/projects/mricron/). The converted data were imported into the Diffusional Kurtosis Estimator (Version 2.5.1; Medical University of South Carolina Center for Biomedical Imaging, Charleston, South Carolina) for preprocessing, namely spatial smoothing (full width at half maximum $=3.75$ ), median filtering, linear trend removal, and denoise processing.

Seven DKI parameters were extracted (Fig 1): mean kurtosis $(\mathrm{MK})$, axial kurtosis (AK), radial kurtosis (RK), fractional anisotropy (FA), mean diffusivity (MD), axial diffusivity $(\mathrm{AD})$, and radial diffusivity $(\mathrm{RD})$. The parameter values were 


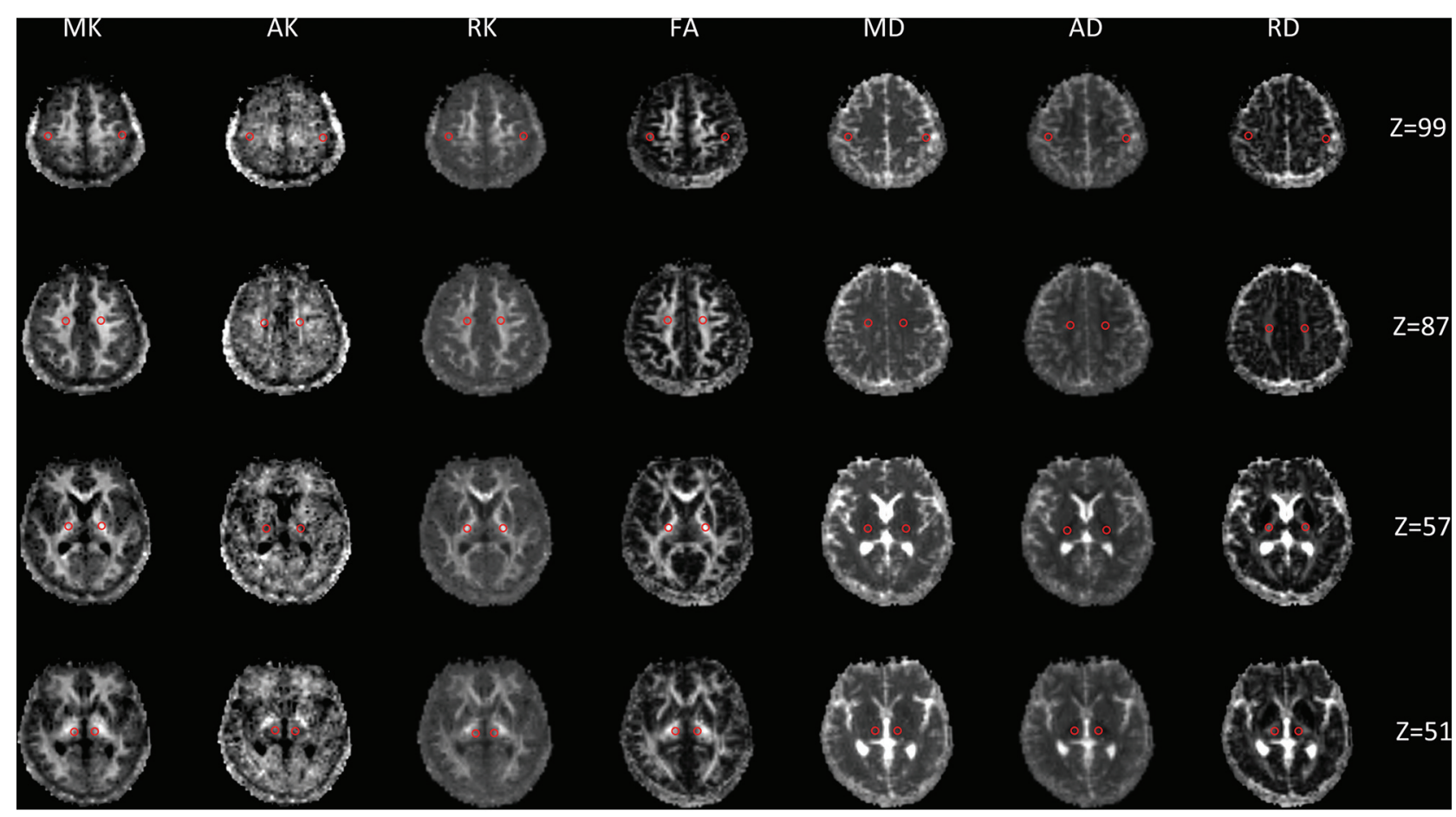

FIG 1. DKI parameter maps of patients with MMD. From left to right: MK, AK, RK, FA, MD, AD, and RD. Selection of ROIs in the bilateral primary $\mathrm{SI}$, corona radiata, posterior limb of the internal capsule, and thalamus is shown in red circles.

measured in the bilateral SI, corona radiata, posterior limb of the internal capsule, and thalamus of all subjects using the ROI method by a radiologist who had $>10$ years of experience in neuroimaging. Three circular ROIs $(5 \mathrm{~mm}$ in diameter) were selected at random from different layers of each brain region. The ROIs were not selected from the infarct region. Finally, the mean of the ROIs was taken as the quantitative DKI parameter value in each region.

\section{DSC-MR Image Analysis}

The DSC-MR images were imported into the syngo via 20 postprocessing workstation (Siemens). Four hemodynamic parameter maps were obtained using the arterial input function model: relative cerebral blood volume, relative cerebral blood flow, relative mean transit time, and time-to-peak (TTP). In clinical practice, we found that TTP is more sensitive than the other 3 parameters in detecting hemodynamic impairment in patients with Moyamoya disease and their hemodynamic improvement after vascular reconstruction. In a study by Wityk et al, ${ }^{15}$ TTP was also used to evaluate hemodynamic changes in patients with Moyamoya disease after vascular reconstruction. Therefore, we selected TTP to evaluate hemodynamic changes in patients with Moyamoya disease. The TTP was measured in the middle cerebral artery supply area surrounding the bilateral central sulcus $(\mathrm{M})$, bilateral corona radiata $(\mathrm{C})$, bilateral basal ganglia (B), bilateral thalamus $(\mathrm{T})$, and bilateral cerebellar hemisphere. The difference in TTP between each region and the ipsilateral cerebellar hemisphere (ie, $\Delta \mathrm{TTP}_{\mathrm{M}}, \Delta \mathrm{TTP}_{\mathrm{C}}, \Delta \mathrm{TTP}_{\mathrm{B}}$, and $\Delta \mathrm{TTP}_{\mathrm{T}}$ ) represents the quantitative parameter of blood perfusion in the bilateral SI, corona radiata, basal ganglia, and thalamus.

\section{Statistical Analysis}

A preliminary comparison revealed no obvious differences in DKI parameters of the bilateral SI, corona radiata, posterior limb of the internal capsule, and thalamus among healthy volunteers. Hence, data from the left and right sides of healthy volunteers were combined to compose the control group. In addition, data from the left and right sides of patients with MMD without acroparesthesia were combined to form the group without acroparesthesia, and data from the symptomatic and asymptomatic sides of patients with unilateral acroparesthesia were combined to form the symptomatic and asymptomatic groups, respectively.

All statistical analyses were performed using SAS (Version 9.3; SAS Institute, Cary, North Carolina). The Kruskal-Wallis test was used to determine differences in DKI parameters among the 4 groups. The paired-samples $t$ test or signed rank test was used to compare DKI parameters between the symptomatic and asymptomatic groups of patients with MMD with acroparesthesia, as well as to compare DKI parameters and $\triangle \mathrm{TTP}$ on the operated side of surgical patients before and 3-4 months after EDAS. A $P$ value $<.05$ was considered statistically significant.

\section{RESULTS}

\section{Differences in DKI Parameters of Sensory Pathway- Related Brain Regions in Patients with MMD}

Comparison of DKI parameters among the 4 groups revealed no significant changes in the SI of patients with MMD compared with healthy volunteers (Fig 2). However, patients with MMD had decreased $M K, A K, R K$, and FA values and increased MD, $\mathrm{AD}$, and $\mathrm{RD}$ values in the corona radiata (Fig 3). In addition, the $\mathrm{MK}, \mathrm{RK}$, and FA decreased, whereas the $\mathrm{MD}$ and $\mathrm{RD}$ values increased in the posterior limb of the internal capsule among 

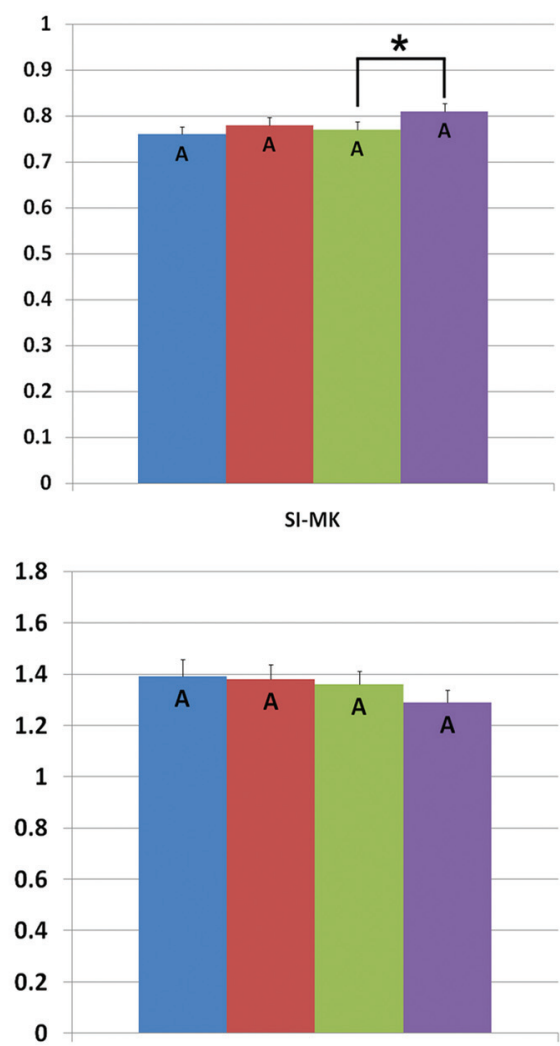

SI-MD

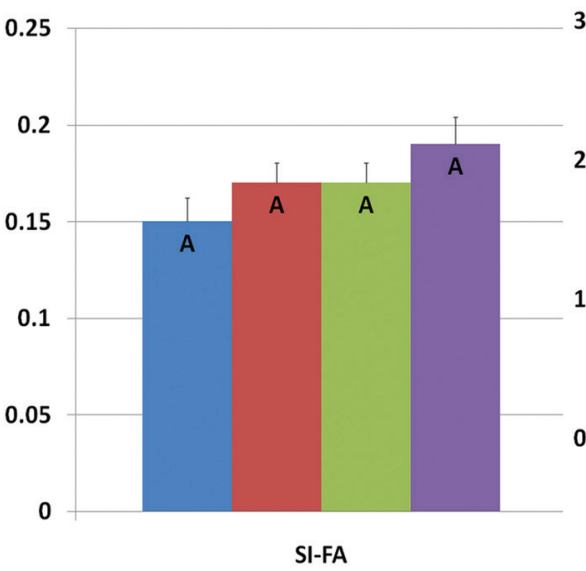

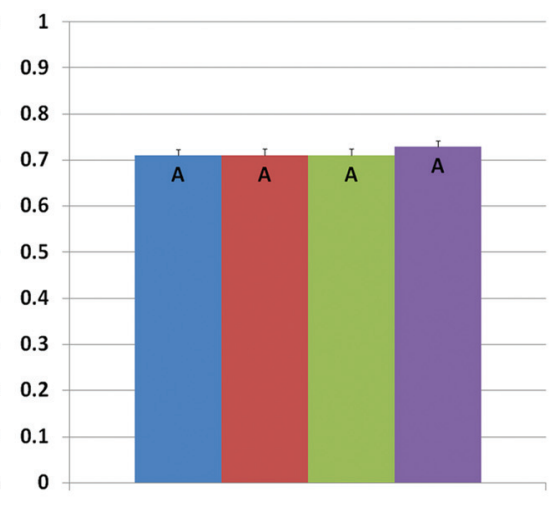

SI-AK
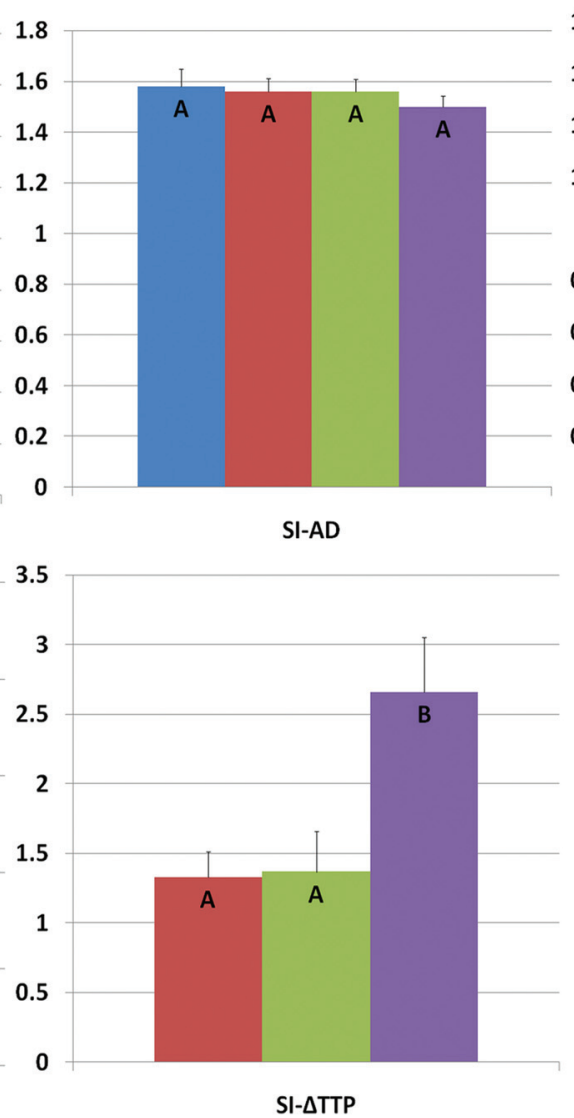

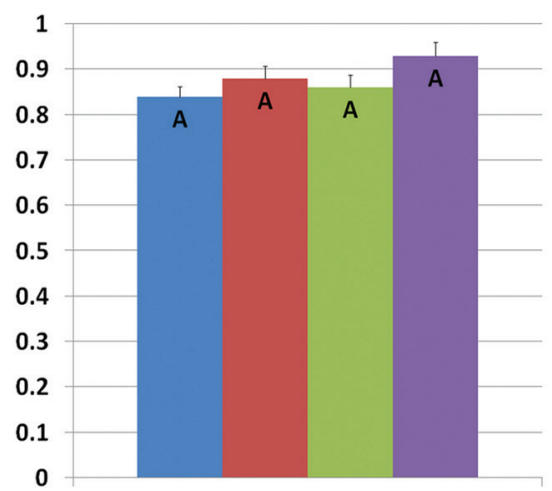

SI-RK

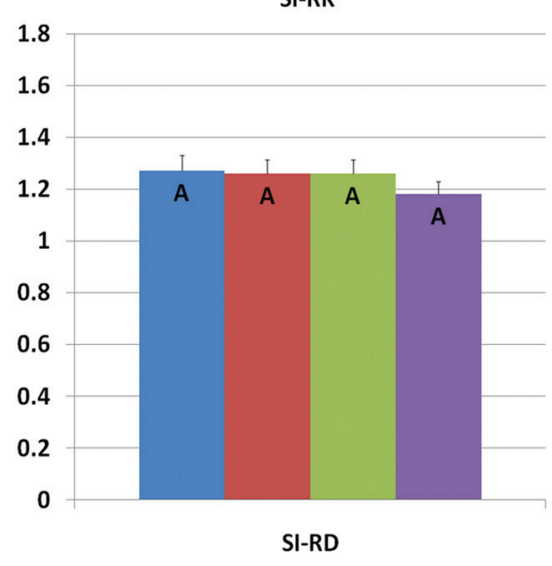

Controls

Patients without paresthesia

Asymptomatic side of patients with paresthesia

Symptomatic side of patients with paresthesia

FIG 2. Comparison of DKI parameters in the contralateral SI among healthy volunteers (controls), patients with MMD without acroparesthesia, the asymptomatic side of patients with MMD with acroparesthesia, and the symptomatic side of patients with MMD with acroparesthesia. Group means were compared using the Kruskal-Wallis test. Different letters on the column indicate a significant difference by pair-wise comparison $(P<.05)$. The asterisk represents a significant difference between the symptomatic and asymptomatic sides of patients with acroparesthesia by self-paired comparison (signed rank test, $P<.05$ ). Error bars represent standard errors.

patients with MMD (Fig 4). Decreased AK and increased RK, $\mathrm{AD}$, and $\mathrm{RD}$ values were noted in the thalami of patients with MMD (Fig 5).

Self-paired comparison of patients with MMD with acroparesthesia revealed that compared with parameter values of the asymptomatic contralateral hemisphere, the MK value was increased and the $\triangle \mathrm{TTP}$ was prolonged in the symptomatic contralateral SI (Fig 2). In addition, increased FA values and prolonged $\triangle \mathrm{TTP}$ were observed in the symptomatic contralateral corona radiata (Fig 3) and posterior limb of the internal capsule (Fig 4). The $\mathrm{AK}$ decreased whereas the $\mathrm{MD}$ and $\mathrm{AD}$ values increased in the symptomatic contralateral thalamus, but no significant change was found in the $\Delta \mathrm{TTP}$ (Fig 5).

\section{Changes in DKI Parameters of Sensory Pathway-Related Brain Regions in Patients with MMD following EDAS}

Among the 15 surgical patients with acroparesthesia, limb symptoms disappeared in 9 patients and were markedly mitigated in the other 6 patients 3-4 months after EDAS. Compared with before the operation, the $\triangle \mathrm{TTP}$ was shortened and the MK value decreased, whereas the MD, $\mathrm{AD}$, and $\mathrm{RD}$ values increased in the SI of the operated side (Table 1). 

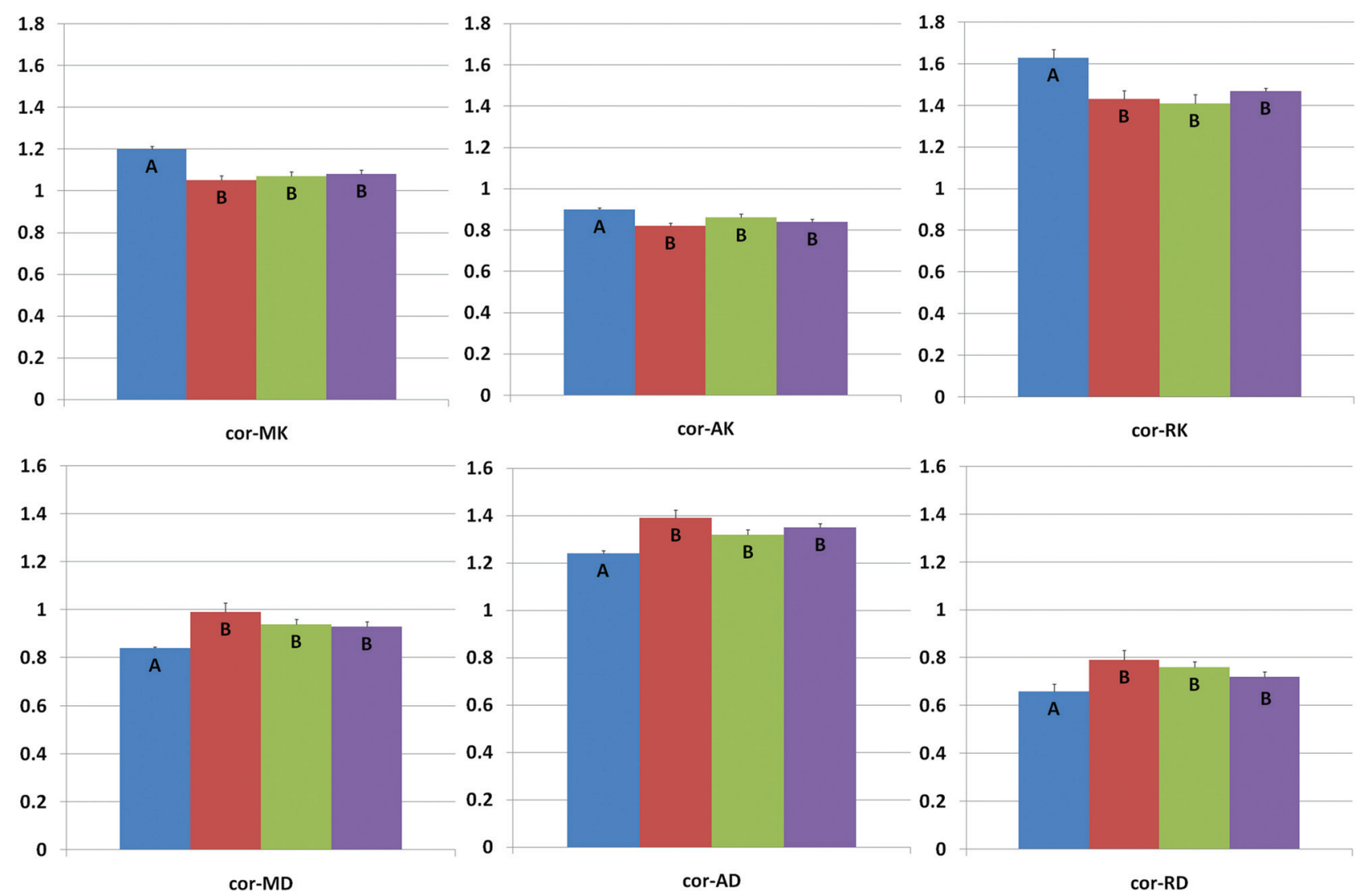

cor-AK

cor-RK
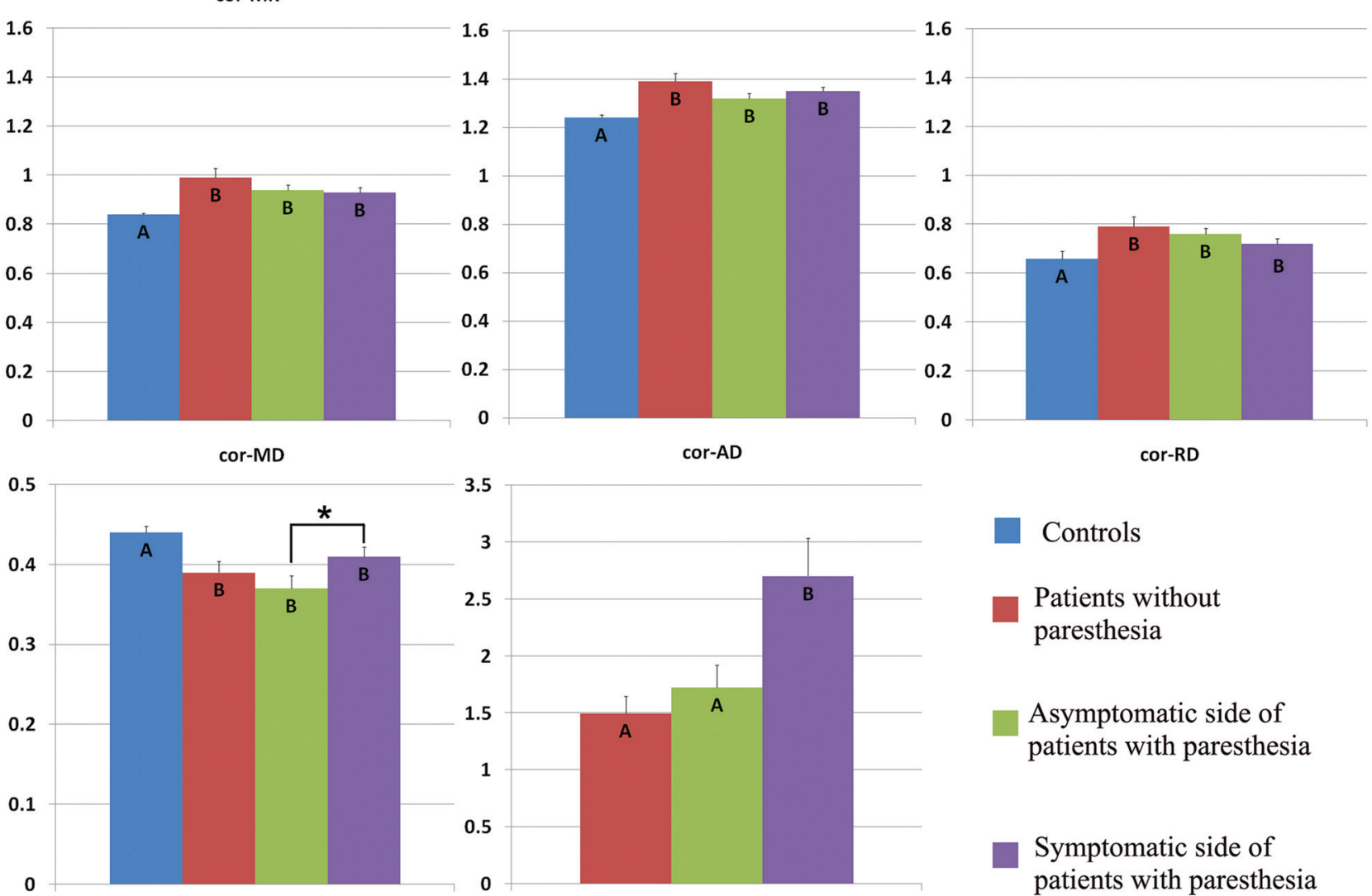

cor-FA

cor- $\triangle T T P$

FIG 3. Comparison of DKI parameters in the contralateral corona radiata among healthy volunteers (controls), patients with MMD without acroparesthesia, the asymptomatic side of patients with MMD with acroparesthesia, and the symptomatic side of patients with MMD with acroparesthesia. Group means were compared using the Kruskal-Wallis test. Different letters on the column indicate a significant difference by pair-wise comparison $(P<.05)$. The asterisk represents a significant difference between the symptomatic and asymptomatic sides of patients with acroparesthesia by self-paired comparison (signed rank test, $P<.05$ ). Error bars represent standard errors. Cor indicates coronal radiata.

However, no significant changes in DKI parameters were observed in the corona radiata, posterior limb of the internal capsule, or thalamus on the operated side.

The preoperative symptoms of the 14 surgical patients without acroparesthesia were dizziness, headache, blurred vision, or inarticulateness. These symptoms were mitigated or disappeared 3-4 months after EDAS. Compared with before the operation, the $\triangle$ TTP was shortened and the MK decreased in the SI on the operated side (Table 2), whereas the $\triangle \mathrm{TTP}$ was shortened, the FA decreased, and the $\mathrm{RD}$ increased in the corona radiata on the operated side (Table 3). The DKI parameters did not change significantly in the posterior limb of the internal capsule or thalamus before and after the operation.

\section{DISCUSSION}

This study shows that DKI can detect microstructural changes in brain tissue among patients with MMD before the detection of brain parenchymal changes by conventional MR imaging. The white matter microstructure may change in patients with MMD during long-term chronic ischemia, even in those with normalappearing white matter. ${ }^{6}$ On the basis of DTI, Jeong et al ${ }^{10}$ found 


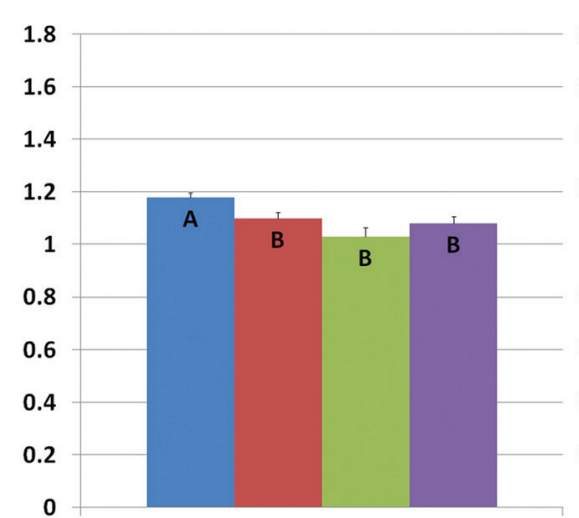

IC-MK
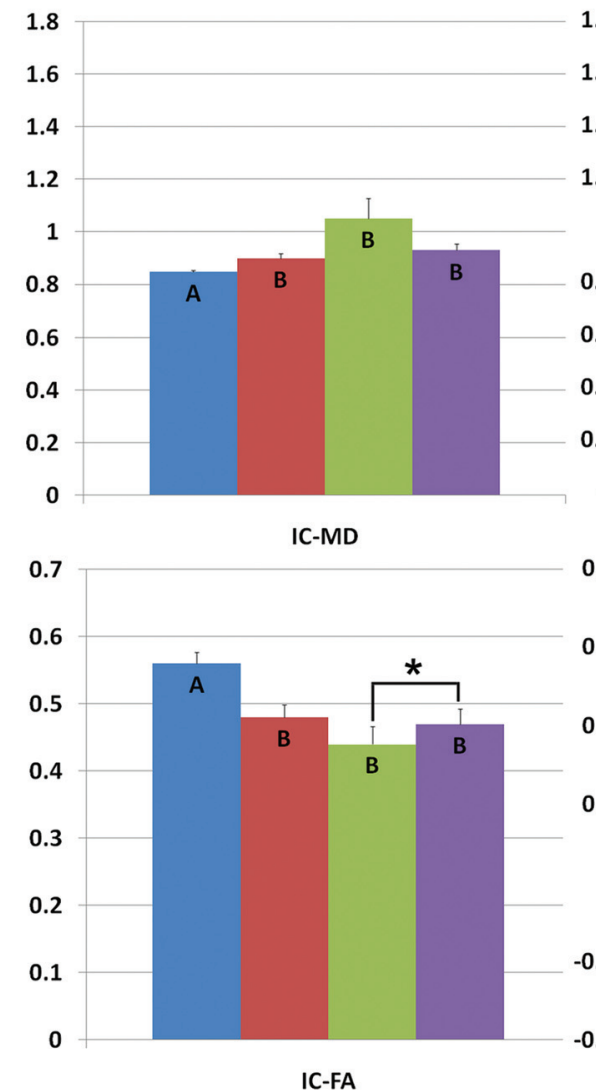

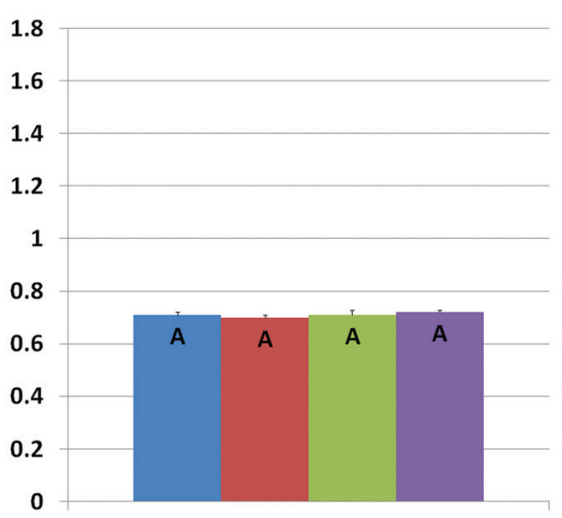

IC-AK

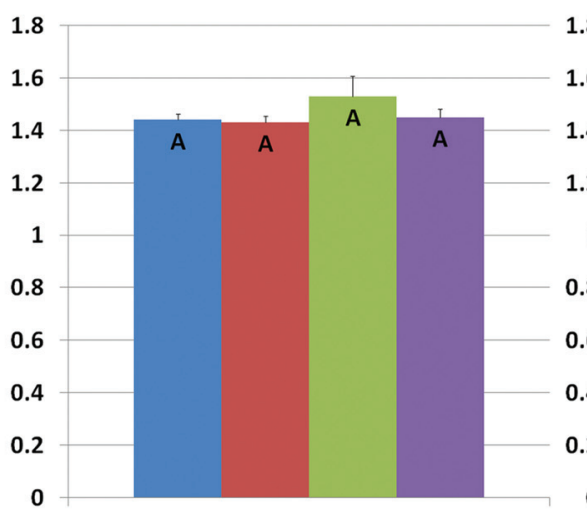

IC-AD

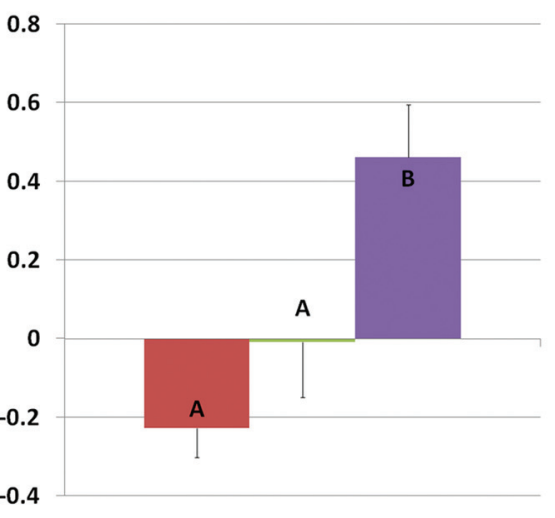

BG- $\triangle$ TTP

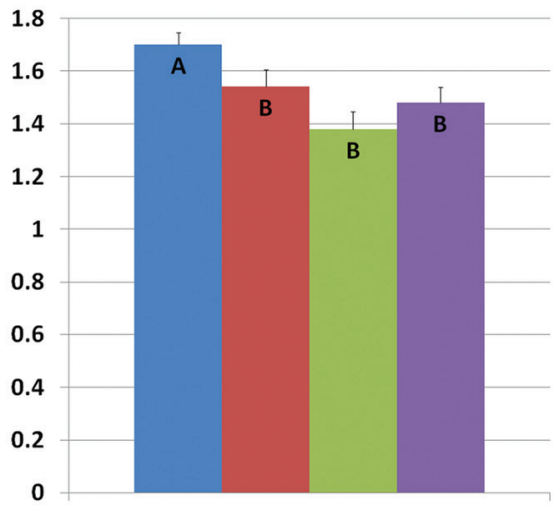

IC-RK

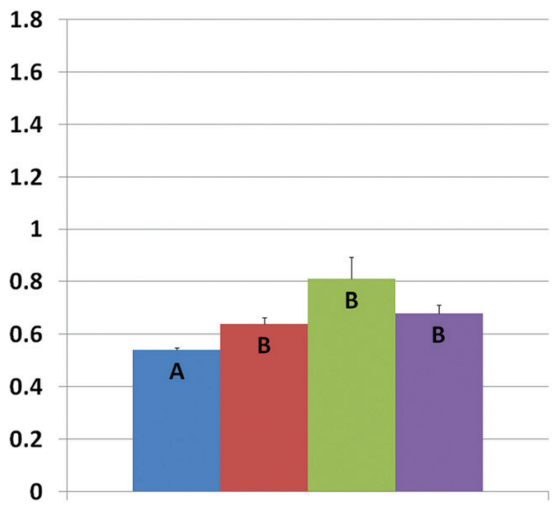

IC-RD

\section{Controls}

Patients without paresthesia

Asymptomatic side of patients with paresthesia

Symptomatic side of patients with paresthesia

FIG 4. Comparison of DKI parameters in the contralateral posterior limb of the internal capsule among healthy volunteers (controls), patients with MMD without acroparesthesia, the asymptomatic side of patients with MMD with acroparesthesia, and the symptomatic side of patients with MMD with acroparesthesia. Group means were compared using the Kruskal-Wallis test. Different letters on the column indicate significant differences by pair-wise comparison $(P<.05)$. The asterisk represents a significant difference between the symptomatic and asymptomatic sides of patients with acroparesthesia by self-paired comparison (signed rank test, $P<.05$ ). Error bars represent standard errors. IC indicates internal capsule.

a decrease in the FA values but an increase in the ADC values in the bilateral corona radiata of patients with MMD. Using DKI, Kazumata et $\mathrm{al}^{16}$ found that the MK value decreased in the white matter on the right forehead; the white matter in the occipital subcortex; and the frontoparietal white matter in the superior longitudinal fasciculus branches, corona radiata, corpus callosum, and bilateral thalamus of patients with MMD. However, these 2 studies did not report microstructural changes in relevant brain regions among patients with MMD with specific clinical symptoms. In other words, the changes observed in the white matter of patients with MMD may occur commonly in long-term chronic ischemic conditions. Therefore, such changes cannot explain the diversity of clinical symptoms in patients with MMD.

Here, we observed the changes in DKI parameters of sensory pathway-related brain regions among patients with MMD with unilateral acroparesthesia. Compared with healthy volunteers, the patients with MMD presented similar changes in sensory pathway-related white matter regions, including the posterior limb of the internal capsule and corona radiata. This result is in agreement with the observations of Jeong et $\mathrm{al}^{10}$ and 

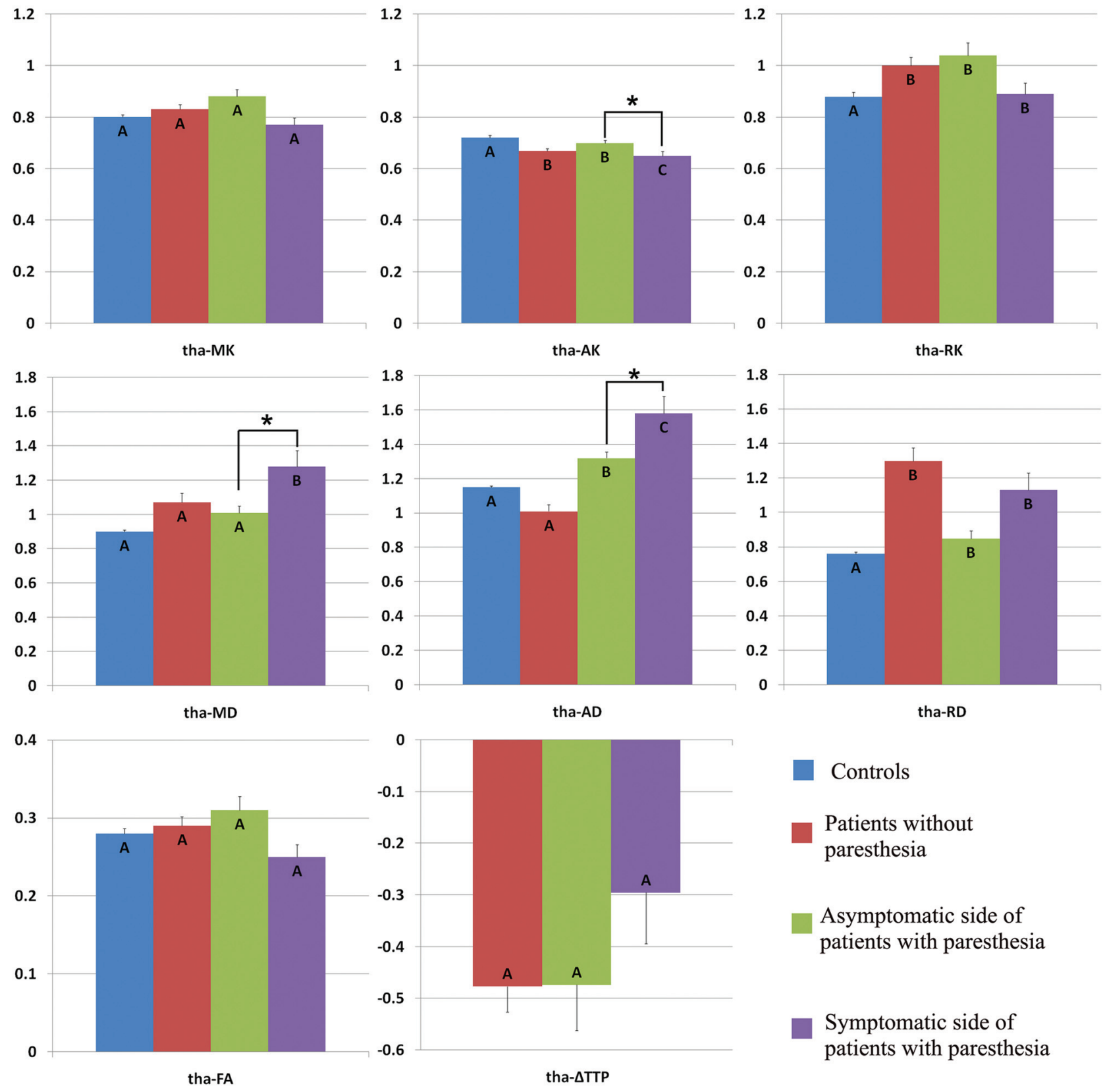

Controls

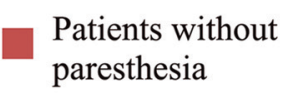

Asymptomatic side of patients with paresthesia

Symptomatic side of patients with paresthesia

FIG 5. Comparison of DKI parameters in the contralateral thalamus among healthy volunteers (controls), patients with MMD without acroparesthesia, the asymptomatic side of patients with MMD with acroparesthesia, and the symptomatic side of patients with MMD with acroparesthesia. Group means were compared using the Kruskal-Wallis test. Different letters on the column indicate a significant difference by pairwise comparison $(P<.05)$. The asterisk represents a significant difference between the symptomatic and asymptomatic sides of patients with acroparesthesia by self-paired comparison (signed rank test, $P<.05$ ). Error bars represent standard errors. Tha indicates thalamus.

Kazumata et al. ${ }^{16}$ Among the DKI parameters reflecting white matter changes, a decrease of RK is associated with demyelination, whereas a change of AK reflects axonal degeneration. ${ }^{17}$ An increase of RD is linked to myelin degeneration or increased extracellular fluid around the ventricle, ${ }^{18}$ and an increase of AD is related to axonal degeneration. ${ }^{19}$ Therefore, dynamic changes in DKI parameters of the white matter among patients with MMD represent degenerative changes in brain tissue during long-term chronic ischemia.

On the basis of paired comparison of sensory pathway-related brain regions between the symptomatic and asymptomatic groups of patients with MMD with acroparesthesia, we observed increased MK values in the contralateral SI, increased FA in the contralateral corona radiata, increased FA values in the contralateral posterior limb of the internal capsule, and increased TTP in the corresponding region of the symptomatic contralateral hemisphere. In an ischemic state, the increase of MK may represent acute brain damage related to axon beading, microglia upregulation, or other microstructural changes. ${ }^{20-22}$ The increase of FA in white matter can occur in the case of insufficient brain perfusion (but not infarction). ${ }^{23,24}$ This change of FA, which also represents acute brain damage, may be related to cytotoxic 
Table 1: Comparison of DKI parameters in the primary somatosensory cortex of the operated side in patients with MMD with unilateral acroparesthesia before and after encephaloduroarteriosynangiosis ${ }^{a}$

\begin{tabular}{|c|c|c|c|c|}
\hline \multirow[b]{2}{*}{ DKI Parameter } & \multicolumn{2}{|c|}{ Primary Somatosensory Cortex } & \multirow[b]{2}{*}{ Statistic } & \multirow[b]{2}{*}{$P$} \\
\hline & Before EDAS & After EDAS & & \\
\hline MK & $0.80 \pm 0.09$ & $0.75 \pm 0.09$ & $t=-2.5012$ & 0.02 \\
\hline AK & $0.72 \pm 0.07$ & $0.68 \pm 0.07$ & $t=-2.1028$ & 0.06 \\
\hline RK & $0.91 \pm 0.15$ & $0.86 \pm 0.16$ & $t=-1.6657$ & 0.12 \\
\hline FA & $0.18 \pm 0.07$ & $0.18 \pm 0.11$ & $S=-15.5$ & 0.30 \\
\hline MD & $1.32 \pm 0.23$ & $1.49 \pm 0.35$ & $t=2.5373$ & 0.02 \\
\hline$A D$ & $1.53 \pm 0.20$ & $1.68 \pm 0.33$ & $t=2.4626$ & 0.03 \\
\hline RD & $1.22 \pm 0.24$ & $1.38 \pm 0.36$ & $t=2.4966$ & 0.02 \\
\hline$\Delta \mathrm{TTP}$ (sec) & $2.55 \pm 2.14$ & $1.07 \pm 0.92$ & $S=46$ & 0.007 \\
\hline
\end{tabular}

Note: $-S$ indicates the Rank Statistic of signed rank sum test.

${ }^{a}$ EDAS; $n=15, \bar{x} \pm \mathrm{s}$. Statistical analysis was performed by a self-paired $t$ test or signed rank sum test.

Table 2: Comparison of DKI parameters in the primary somatosensory cortex of the operated side in patients with MMD without acroparesthesia before and after encephaloduroarteriosynangiosis ${ }^{a}$

\begin{tabular}{lcccc}
\hline & \multicolumn{2}{c}{ Primary Somatosensory Cortex } & & \\
\cline { 2 - 3 } DKI Parameter & Before EDAS & After EDAS & Statistic & $\boldsymbol{P}$ \\
\hline MK & $0.81 \pm 0.11$ & $0.75 \pm 0.11$ & $t=-2.3113$ & 0.04 \\
AK & $0.73 \pm 0.10$ & $0.70 \pm 0.09$ & $t=-1.1748$ & 0.26 \\
RK & $0.92 \pm 0.17$ & $0.83 \pm 0.19$ & $t=-2.1186$ & 0.05 \\
FA & $0.18 \pm 0.07$ & $0.15 \pm 0.07$ & $t=-2.009$ & 0.06 \\
MD & $1.31 \pm 0.30$ & $1.43 \pm 0.40$ & $S=18$ & 0.32 \\
AD & $1.50 \pm 0.29$ & $1.61 \pm 0.39$ & $S=18.5$ & 0.36 \\
RD & $1.17 \pm 0.29$ & $1.33 \pm 0.41$ & $S=22.5$ & 0.21 \\
$\Delta$ TTP (sec) & $2.67 \pm 1.52$ & $0.93 \pm 0.76$ & $S=-51.5$ & $<0.001$ \\
\hline
\end{tabular}

Note: $-S$ indicates the Rank Statistic of signed rank sum test.

${ }^{a}$ EDAS; $n=14, \bar{x} \pm \mathrm{s}$. Statistical analysis was performed by a self-paired $t$ test or signed rank sum test.

Table 3: Comparison of DKI parameters in the corona radiata of the operated side in patients with MMD without acroparesthesia before and after encephaloduroarteriosynangiosis ${ }^{\mathrm{a}}$

\begin{tabular}{|c|c|c|c|c|}
\hline \multirow[b]{2}{*}{ DKI Parameter } & \multicolumn{2}{|c|}{ Corona Radiata } & \multirow[b]{2}{*}{ Statistic } & \multirow[b]{2}{*}{$P$} \\
\hline & Before EDAS & After EDAS & & \\
\hline MK & $1.11 \pm 0.09$ & $1.06 \pm 0.12$ & $t=-1.41963$ & 0.17 \\
\hline AK & $0.84 \pm 0.06$ & $0.83 \pm 0.05$ & $t=-1.20239$ & 0.25 \\
\hline RK & $1.54 \pm 0.25$ & $1.37 \pm 0.21$ & $t=-1.97407$ & 0.07 \\
\hline FA & $0.42 \pm 0.09$ & $0.35 \pm 0.07$ & $S=-39$ & 0.04 \\
\hline MD & $0.91 \pm 0.08$ & $0.95 \pm 0.09$ & $S=31$ & 0.08 \\
\hline$A D$ & $1.35 \pm 0.11$ & $1.30 \pm 0.09$ & $t=1.7969$ & 0.09 \\
\hline $\mathrm{RD}$ & $0.70 \pm 0.11$ & $0.77 \pm 0.10$ & $S=43$ & 0.04 \\
\hline$\Delta \mathrm{TTP}(\mathrm{s})$ & $2.13 \pm 1.29$ & $1.73 \pm 1.23$ & $S=48$ & 0.02 \\
\hline
\end{tabular}

Note: $-S$ indicates the Rank Statistic of signed rank sum test.

${ }^{a}$ EDAS; $n=14, \bar{x} \pm \mathrm{s}$. Statistical analysis was performed by a self-paired $t$ test or signed rank sum test.

edema without a significant change in structural coherence. ${ }^{25}$ All such brain damage may be the main cause of the clinical symptoms in patients with MMD. Among the patients with MMD undergoing revascularization, the $\triangle \mathrm{TTP}$ was shortened and the MK value was decreased in the SI on the operated side. In addition, the $\triangle \mathrm{TTP}$ was shortened, and the FA value decreased in the corona radiata on the operated side. All these postoperative changes were associated with improved clinical symptoms in the surgical patients. These results suggest that the observed changes in the SI and white matter regions are reversible along with the improvement of cerebral blood perfusion.
This study was the first to investigate changes in DKI parameters of sensory pathway-related brain regions among patients with MMD with acroparesthesia. In the case of longterm chronic ischemia, the microstructure of brain tissue may be altered and the DKI parameters likely undergo changes (a decrease in kurtosis, a decrease in FA, and an increase in diffusivity) in patients with MMD. When the hemodynamic state is stable, these parameters are also in a relatively stable or pseudonormal state; on further hemodynamic damage, some acute or specific changes may occur in the microstructure of the brain, causing DKI parameter changes (an increase in the MK of the gray matter and an increase in the FA of the white matter). These changes would return to a pseudonormal state during hemodynamic improvement after revascularization.

During the follow-up of MMD, DSC-MR imaging is an important technique for the assessment of cerebral blood perfusion; however, there is no clear standard to assess the severity of the disease. Our results indicate that DKI enables timely detection of changes in brain tissue microstructure of patients with MMD with reduced cerebral blood perfusion, which is helpful to assess the disease severity and the surgical outcome.

Our study has some limitations. First, the patients had re-examination results for only 3-4 months after the operation and lacked a long-term follow-up record. Because indirect revascularization was used as the surgical approach for all patients, a longer follow-up period could facilitate the study of changes in DKI parameters during hemodynamic improvement. Second, due to the small sample size, especially of postoperative samples, it was impossible to effectively determine changes in DKI parameters for patients with different symptom improvements after the operation. Third, functional imaging techniques that can quantitatively reflect cerebral blood flow changes, such as PET and SPECT, were not used for the hemodynamic assessment.

\section{CONCLUSIONS}

The DKI technique can detect microstructural changes in the cerebral white and gray matter of patients with MMD before and after revascularization in a timely manner. It is a sensitive 
method for use in the follow-up of patients with MMD, which supports the assessment of disease severity and surgical outcome.

Disclosures: Peng-Gang Qiao-RELATED: Grant: National Natural Science Foundation of China, Comments: grant No. 81701663. Money paid to the institution.

\section{REFERENCES}

1. Research Committee on the Pathology and Treatment of Spontaneous Occlusion of the Circle of Willis, Health Labour Sciences Research Grant for Research on Measures for Infractable Diseases. Guidelines for diagnosis and treatment of moyamoya disease (spontaneous occlusion of the circle of Willis). Neurol Med Chir (Tokyo) 2012; 52:245-66 CrossRef Medline

2. Suzuki J, Takaku A. Cerebrovasular "moyamoya” disease: disease showing abnormal net-like vessels in base of brain. Arch Neurol 1969;20:288-99 CrossRef Medline

3. Hervé D, Kossorotoff M, Bresson D, et al. French clinical practice guidelines for Moyamoya angiopathy. Rev Neurol (Paris) 2018;174: 292-303 CrossRef Medline

4. Ikeda K, Iwasaki Y, Kashihara H, et al. Adult moyamoya disease in the asymptomatic Japanese population. J Clin Neurosci 2006;13: 334-38 CrossRef Medline

5. Kuroda S, Hashimoto N, Yoshimoto T, et al; Research Committee on Moyamoya Disease in Japan. Radiological findings, clinical course, and outcome in asymptomatic moyamoya disease: results of multicenter survey in Japan. Stroke 2007;38:1430-35 CrossRef Medline

6. Hara S, Hori M, Murata S, et al. Microstructural damage in normalappearing brain parenchyma and neurocognitive dysfunction in adult Moyamoya disease. Stroke 2018;49:2504-07 CrossRef Medline

7. Farrell JA, Zhang J, Jones MV, et al. Q-space and conventional diffusion imaging of axon and myelin damage in the rat spinal cord after axotomy. Magn Reson Med 2010;63:1323-35 CrossRef Medline

8. Jiang Q, Qu C, Chopp M, et al. MRI evaluation of axonal reorganization after bone marrow stromal cell treatment of traumatic brain injury. NMR Biomed 2011;24:1119-28 CrossRef Medline

9. Takagi T, Nakamura M, Yamada M, et al. Visualization of peripheral nerve degeneration and regeneration: monitoring with diffusion tensor tractography. Neuroimage 2009;44:884-92 CrossRef Medline

10. Jeong H, Kim J, Choi HS, et al. Changes in integrity of normalappearing white matter in patients with moyamoya disease: a diffusion tensor imaging study. AJNR Am J Neuroradiol 2011;32:189398 CrossRef Medline

11. Bandettini PA, Wong EC, Hinks RS, et al. Time course EPI of human brain function during task activation. Magn Reson Med 1992;25:390-97 CrossRef Medline
12. Raab P, Hattingen E, Franz K, et al. Cerebral gliomas: diffusional kurtosis imaging analysis of microstructural differences. Radiology 2010;254:876-81 CrossRef Medline

13. Jensen $\mathrm{JH}$, Falangola $\mathrm{MF}, \mathrm{Hu} \mathrm{C}$, et al. Preliminary observations of increased diffusional kurtosis in human brain following recent cerebral infarction. NMR Biomed 2011;24:452-57 CrossRef Medline

14. Hui ES, Fieremans E, Jensen JH, et al. Stroke assessment with diffusional kurtosis imaging. Stroke 2012;43:2968-73 CrossRef Medline

15. Wityk RJ, Hillis A, Beauchamp N, et al. Perfusion-weighted magnetic resonance imaging in adult moyamoya syndrome: characteristic patterns and change after surgical intervention: case report. Neurosurgery 2002;51:1499-505 Medline

16. Kazumata K, Tha KK, Narita $\mathrm{H}$, et al. Characteristics of diffusional kurtosis in chronic ischemia of adult moyamoya disease: comparing diffusional kurtosis and diffusion tensor imaging. AJNR Am J Neuroradiol 2016;37:1432-39 CrossRef Medline

17. Cheung MM, Hui ES, Chan KC, et al. Does diffusion kurtosis imaging lead to better neural tissue characterization? A rodent brain maturation study. Neuroimage 2009;45:386-92 CrossRef Medline

18. Cechetti F, Pagnussat AS, Worm PV, et al. Chronic brain hypoperfusion causes early glial activation and neuronal death, and subsequent long-term memory impairment. Brain Res Bull 2012;87:10916 CrossRef Medline

19. Kazumata K, Tha KK, Narita H, et al. Chronic ischemia alters brain microstructural integrity and cognitive performance in adult Moyamoya disease. Stroke 2015;46:354-60 CrossRef Medline

20. Weber RA, Hui ES, Jensen JH, et al. Diffusional kurtosis and diffusion tensor imaging reveal different time-sensitive stroke-induced microstructural changes. Stroke 2015;46:545-50 CrossRef Medline

21. Zhu LH, Zhang ZP, Wang FN, et al. Diffusion kurtosis imaging of microstructural changes in brain tissue affected by acute ischemic stroke in different locations. Neural Regen Res 2019;14:272-79 CrossRef Medline

22. Guo YL, Zhang ZP, Zhang GS, et al. Evaluation of mean diffusion and kurtosis MRI mismatch in subacute ischemic stroke: comparison with NIHSS score. Brain Res 2016;1644:231-39 CrossRef Medline

23. Nael K, Trouard TP, Lafleur SR, et al. White matter ischemic changes in hyperacute ischemic stroke: voxel-based analysis using diffusion tensor imaging and MR perfusion. Stroke 2015;46:413-18 CrossRef Medline

24. Carano RA, Li F, Irie K, et al. Multispectral analysis of the temporal evolution of cerebral ischemia in the rat brain. J Magn Reson Imaging 2000;12:842-58 CrossRef Medline

25. Yang Q, Tress BM, Barber PA, et al. Serial study of apparent diffusion coeffcient and anisotropy in patients with acute stroke. Stroke 1999;30:2382-90 CrossRef Medline 